Bull. Austral. Math. Soc.

$42 \mathrm{~B} 20,42 \mathrm{C} 10$

VOL. 75 (2007) [397-408]

\title{
WEAK TYPE ESTIMATES FOR RIESZ-LAGUERRE TRANSFORMS
}

\author{
Emanuela Sasso
}

\begin{abstract}
We prove that the first order Riesz transforms associated to the Laguerre semigroup are weak-type $(1,1)$. We also present a counterexample showing that for the Riesz transforms of order three or higher the weak type $(1,1)$ estimate fails.
\end{abstract}

\section{INTRODUCTION}

The aim of this paper is to study the weak type $(1,1)$ boundedness of the Riesz transform $\mathcal{R}_{\alpha}$ naturally associated with the multidimensional Laguerre operator $\mathcal{L}_{\alpha}$, whenever $\alpha=\left(\alpha_{1}, \ldots, \alpha_{d}\right)$ is a multi-index with $\alpha_{i} \geqslant 0, i=1, \ldots, d$ (see Section 2 for all unexplained terminology and notation).

Riesz transforms and conjugate Poisson integrals for the Laguerre semigroup were first studied by Muckenhoupt [6] in the one dimensional case. The $L^{p}$ boundedness, with $1<p<\infty$, was obtained by Nowak in multidimensional case [7]. By an analytic method based on Littlewood-Paley-Stein theory, he extended the previous results of Gutiérrez, Incognito and Torrea [5], true only for a discrete set of half-integer multi-indices $\alpha$. Recently Graczyk, Loeb, Lopez, Nowak and Urbina [4] obtained the $L^{p}$-boundedness of the Riesz-Laguerre transforms for any order and the weak type $(1,1)$ when the order is equal to 2. The corresponding proof is based on the technique of transference from the Hermite setting, and therefore only half-integer type multi-indices $\alpha$ are considered.

This paper complements the analysis of the first order Riesz-Laguerre transforms. In particular, this paper is basically devoted to the proof of the following main result.

THEOREM 1.1. The first order Riesz-Laguerre transforms $\mathcal{R}_{\alpha}$ are of weak type $(1,1)$ with respect to the Laguerre measure, for each $\alpha \in(0, \infty)^{d}$.

Furthermore, we shall give a counterexample for the weak type $(1,1)$ unboundedness for order three and higher.

Observe that the natural range of $\alpha$ for the Laguerre setting is $(-1, \infty)^{d}$. The restriction to $\alpha \in(0, \infty)^{d}$ is imposed by methods used. Moreover transforms of order 2 are not treated, but we may conjecture the weak type $(1,1)$ for these operators. In fact, this result was proved recently in [4] for half-integer $\alpha$.

Received 30th October, 2006

Copyright Clearance Centre, Inc. Serial-fee code: 0004-9727/07 \$A2.00+0.00. 
As in [2] and [5], our starting point is the relationship between Laguerre and Ornstein-Uhlenbeck semigroups. Indeed, it is well known that for half-integer values of the parameter $\alpha$, the Laguerre semigroup can be interpreted as the Ornstein-Uhlenbeck one acting on polyradial functions. Thus we adapt to our case the strategy used in $[3,8,9]$ for the analysis of the Riesz transforms associated to the Ornstein-Uhlebeck operator to obtain the desired results.

The paper is organised as follows. Section 2 contains basic facts and notation needed in the sequel. In particular we determine the distributional kernel of the Riesz-Laguerre transforms and we exploit the relationship with the Ornstein-Uhlenbeck case. The proof of the weak-type $(1,1)$ boundedness of the first order Riesz-Laguerre transform consists of two parts, corresponding to the local and global parts of the operator, is found in Section 3. The analysis of the local part is based on comparison with the Calderón-Zygmund singular integral theory. The reason for this is that "locally" the Laguerre measure is essentially proportional to a polynomial measure; that is, a measure possessing the doubling property. To prove the remaining, we control the global part of the operator by the maximal operator associated to the Laguerre semigroup, which is of weak-type $(1,1)$ $[2,11]$. Finally in Section 4, we present a counterexample, valid in arbitrary dimension, to show that the Riesz-Laguerre transforms of order at least three are not of weak type $(1,1)$ with respect to the Laguerre measure.

\section{THE FIRST ORDER RIESZ-LAGUERRE TRANSFORM}

The Laguerre operator $\mathcal{L}_{\alpha}$ is a self-adjoint "Laplacian" on $L^{2}\left(\mu_{\alpha}\right)$, where $\mu_{\alpha}$ is the Laguerre measure of type $\alpha$ on $\mathbf{R}_{+}^{d}$, that is $\mathrm{d} \mu_{\alpha}(x)=\prod_{i=1}^{d}\left(x_{i}^{\alpha_{i}} e^{-x_{i}}\right) /\left(\Gamma\left(\alpha_{i}+1\right)\right) \mathrm{d} x$ on $\mathbf{R}_{+}^{d}=\left\{x \in \mathbf{R}^{d}: x_{i}>0\right.$, for each $\left.i=1, \ldots, d\right\}$. It is well known that the spectral resolution of $\mathcal{L}_{\alpha}$ is

$$
\mathcal{L}_{\alpha}=\sum_{n=0}^{\infty} n \mathcal{P}_{n}^{\alpha}
$$

where $\mathcal{P}_{n}^{\alpha}$ is the orthogonal projection on the space spanned by Laguerre polynomials of total degree $n$ and type $\alpha$ in $d$ variables (see, for instance, [13]). The operator $\mathcal{L}_{\alpha}$ is the infinitesimal generator of a "heat" semigroup, called the Laguerre semigroup, $\left\{e^{-t \mathcal{L}_{\alpha}}\right.$ : $t \geqslant 0\}$, defined in the spectral sense as

$$
e^{-t \mathcal{L}_{\alpha}}=\sum_{n=0}^{\infty} e^{-n t} \mathcal{P}_{n}^{\alpha}
$$

It can be shown that for each $t>0, e^{-t \mathcal{L}_{\alpha}}$ is an integral operator, whose kernel with respect to the Laguerre measure is

$$
m_{\alpha, t}(x, y)=\left(1-e^{-t}\right)^{-|\alpha|-d} \int_{[-1,1]^{d}} e^{-\left(q_{-}\left(e^{-t / 2} x, y, s\right)\right) /\left(1-e^{-t}\right)} e^{-|y|} \Pi_{\alpha}(s) \mathrm{d} s,
$$


where

$$
\begin{aligned}
q_{ \pm}(x, y, s) & =\sum_{i=1}^{d}\left(x_{i}+y_{i} \pm 2\left(x_{i} y_{i}\right)^{1 / 2} s_{i}\right) \\
\Pi_{\alpha}(s) & =\prod_{i=1}^{d} \frac{\Gamma\left(\alpha_{i}+1\right)}{\Gamma\left(\alpha_{i}+1 / 2\right) \sqrt{\pi}}\left(1-s_{i}^{2}\right)^{\alpha_{i}-1 / 2}
\end{aligned}
$$

and $|\alpha|=\alpha_{1}+\cdots+\alpha_{d}$. See, for instance, [2] and [13]. Indeed, the Laguerre heat kernel is easily computed by means of a classical bilinear generating function for Laguerre polynomials, and then (1) emerges from certain integral representations for the modified Bessel functions of the first kind. The importance of the exact description of the kernel $m_{\alpha, t}$ will be clarified in the following. Indeed, by spectral theory, the Riesz-Laguerre transforms may be written in terms of the Laguerre semigroup.

The first order Riesz-Laguerre transform $\mathcal{R}_{\alpha}=\left(R_{1}^{\alpha}, \ldots, R_{d}^{\alpha}\right)$ is formally defined by

$$
\mathcal{R}_{\alpha}=\nabla_{\alpha}\left(\mathcal{L}_{\alpha}\right)^{-1 / 2} \mathcal{P}_{0}^{\alpha \perp}
$$

where $\nabla_{\alpha}$ is the natural gradient associated to $\mathcal{L}_{\alpha}$, that is, $\nabla_{\alpha}=\left(\sqrt{x_{1}} \partial_{x_{1}}, \ldots, \sqrt{x_{d}} \partial_{x_{d}}\right)$, and $\mathcal{P}_{0}^{\alpha \perp}$ denotes the orthogonal projection onto the orthogonal complement of the eigenspace corresponding to the Laguerre eigenvalue 0 . By spectral theory $\mathcal{R}_{\alpha}$ can be written by means of the Laguerre semigroup. Indeed, using the formula

$$
s^{-b}=\frac{1}{\Gamma(b)} \int_{0}^{\infty} e^{-t s} t^{b} \frac{\mathrm{d} t}{t},
$$

with $b, s>0$ we may define the powers of $\mathcal{L}_{\alpha}$ on $\mathcal{P}_{0}^{\alpha \perp} L^{2}\left(\mu_{\alpha}\right)$ by the formula

$$
\mathcal{L}_{\alpha}^{-b} f=\frac{1}{\Gamma(b)} \int_{0}^{\infty} T_{t}^{\alpha} f t^{b} \frac{\mathrm{d} t}{t}
$$

or equivalently

$$
\mathcal{L}_{\alpha}^{-b} f=\frac{1}{\Gamma(b)} \int_{0}^{1} r^{\mathcal{L}_{\alpha}} f\left(\log \frac{1}{r}\right)^{b-1} \frac{\mathrm{d} r}{r} .
$$

Now we shall deduce by (3) that, off the diagonal, the kernel of $\mathcal{L}_{\alpha}^{-b} \mathcal{P}_{0}^{\alpha \perp}$ with respect to the Laguerre measure agrees with the function $K_{b}$, defined by

$$
K_{b}(x, y)=\frac{1}{\Gamma(b)} \int_{0}^{1}\left(m_{\alpha, \log (1 / r)}(x, y)-1\right)(-\log r)^{b-1} \frac{\mathrm{d} r}{r},
$$

in the sense that for all test functions $f$ and $g$ on $\mathbf{R}_{+}^{d}$, the following identity holds

$$
\left\langle\mathcal{L}_{\alpha}^{-b} \mathcal{P}_{0}^{\alpha \perp} f, g\right\rangle_{\mu_{\alpha}}=\iint K_{b}(x, y) f(y) g(x) \mathrm{d} \mu_{\alpha}(x) \mathrm{d} \mu_{\alpha}(y),
$$


where $\langle\cdot, \cdot\rangle_{\mu_{\alpha}}$ denotes the standard inner product in $L^{2}\left(\mu_{\alpha}\right)$. Indeed, it is not hard to prove (see [3, Lemma 2.2] where this is proved in the Hermite setting) that the above double integral converges absolutely and its absolute value is bounded by $C\|f\|_{\infty}\|g\|_{\infty}$, where $C$ depends on the support of $f$ and $g$. Moreover, by the spectral theorem we may view $\mathcal{L}_{\alpha}^{-b} \mathcal{P}_{0}^{\alpha \perp}$ as the limit of $\left(\varepsilon \mathcal{I}+\mathcal{L}_{\alpha}\right)^{-b} \mathcal{P}_{0}^{\alpha \perp}$ as $\varepsilon$ tends to $0^{+}$. Since the integral kernel of $\left(\varepsilon \mathcal{I}+\mathcal{L}_{\alpha}\right)^{-b} \mathcal{P}_{0}^{\alpha \perp}$ is

$$
J_{\varepsilon, b}(x, y)=\frac{1}{\Gamma(b)} \int_{0}^{1}\left(m_{\alpha, \log \frac{1}{r}}(x, y)-1\right)(-\log r)^{b-1} r^{\varepsilon-1} \mathrm{~d} r,
$$

we only need to show that

$$
\lim _{\varepsilon \rightarrow 0} \iint J_{\varepsilon, b} f(y) g(x) \mathrm{d} \mu_{\alpha}(x) \mathrm{d} \mu_{\alpha}(y)=\iint K_{b}(x, y) f(y) g(x) \mathrm{d} \mu_{\alpha}(x) \mathrm{d} \mu_{\alpha}(y)
$$

for all test functions $f$ and $g$, and this is immediate in view of the absolute convergence of $\iint K_{b}(x, y) f(y) g(x) \mathrm{d} \mu_{\alpha}(x) \mathrm{d} \mu_{\alpha}(y)$.

This allows us to see that the kernel of the Riesz-Laguerre transform $\mathcal{R}_{\alpha}$ coincides with the gradient $\nabla_{\alpha}$ of $K_{1 / 2}$, whenever $x \neq y$, where

$$
\nabla_{\alpha} K_{1 / 2}(x, y)=\left(\sqrt{x_{1}} \partial_{x_{1}} K_{1 / 2}(x, y), \ldots, \sqrt{x_{d}} \partial_{x_{d}} K_{1 / 2}(x, y)\right) \text {. }
$$

As in $[8,9]$ the idea is to decompose $\mathcal{R}_{\boldsymbol{\alpha}}$ into two operators, one given by a kernel supported off the diagonal, and the other satisfying "standard" gradient estimates in a suitable neighbourhood of the diagonal.

To exploit the aforementioned relationship with the Ornstein-Uhlenbeck case, it is convenient to perform a change of coordinates in $\mathbf{R}_{+}^{d}$. If $x=\left(x_{1}, \ldots, x_{d}\right)$ is a vector in $\mathbf{R}_{+}^{d}$, then $x^{2}$ denotes the vector $x^{2}=\left(x_{1}^{2}, \ldots, x_{d}^{2}\right)$. Let $\Psi: \mathbf{R}_{+}^{d} \rightarrow \mathbf{R}_{+}^{d}$ be defined by $\Psi(x)=x^{2}$ and let $\tilde{\mu}_{\alpha}=\mu_{\alpha} \circ \Psi^{-1}$ be the pull-back of measure $\mu_{\alpha}$. Then $\tilde{\mu}_{\alpha}$ is the probability measure

$$
\mathrm{d} \widetilde{\mu}_{\alpha}(x)=2^{d} \prod_{i=1}^{d} \frac{x_{i}^{2 \alpha_{i}+1} e^{-x_{i}^{2}}}{\Gamma\left(\alpha_{i}+1\right)} \mathrm{d} x,
$$

on $\mathbf{R}_{+}^{d}$. The map $f \rightarrow \mathcal{U}_{\Psi} f=f \circ \Psi$ is an isometry of $L^{q}\left(\mu_{\alpha}\right)$ onto $L^{q}\left(\tilde{\mu}_{\alpha}\right)$ and of $L^{q, \infty}\left(\mu_{\alpha}\right)$ onto $L^{q, \infty}\left(\tilde{\mu}_{\alpha}\right)$, for every $q$ in $[1, \infty]$. So we may reduce the problem to the study of the weak type $(1,1)$ boundedness of the operator $\widetilde{\mathcal{R}}_{\alpha}=\mathcal{U}_{\Psi} \mathcal{R}_{\alpha} \mathcal{U}_{\Psi}^{-1}$ with respect to the measure $\tilde{\mu}_{\alpha}$ : Observe that $\tilde{\mathcal{R}}_{\alpha}$ coincides, up to a multiplicative constant, with $\nabla\left(\tilde{\mathcal{L}}_{\alpha}\right)^{-1 / 2} \tilde{\mathcal{P}}_{0}^{\alpha \perp}$, with $\tilde{\mathcal{L}}_{\alpha}=\mathcal{U}_{\Psi} \mathcal{L}_{\alpha} \mathcal{U}_{\Psi}^{-1}, \tilde{\mathcal{P}}_{0}^{\alpha \perp}=\mathcal{U}_{\Psi} \mathcal{P}_{0}^{\alpha \perp} \mathcal{U}_{\Psi}^{-1}$ and $\nabla$ is the gradient on $\mathbf{R}^{d}$ with respect to the Lebesgue measure. In particular, off the diagonal, the operator $\widetilde{\mathcal{R}}_{\alpha}=\left(\widetilde{\mathcal{R}}_{\alpha, 1}, \ldots, \widetilde{\mathcal{R}}_{\alpha, d}\right)$ is given by the smooth kernel $\nabla K_{1 / 2}\left(x^{2}, y^{2}\right)$, that is,

$$
\tilde{\mathcal{R}}_{\alpha, i} f(x)=\int_{\mathbf{R}_{+}^{d}} \partial_{x_{i}} K_{1 / 2}\left(x^{2}, y^{2}\right) f(y) \mathrm{d} \widetilde{\mu}_{\alpha}(y),
$$

for each $i=1, \ldots, d$ and $x \notin \operatorname{supt} f$. 


\section{WEAK-TYPE $(1,1)$ BOUNDEDNESS}

In this section we shall prove the weak type $(1,1)$ boundedness of $\tilde{\mathcal{R}}_{\alpha}$ with respect to the measure $\tilde{\mu}_{\alpha}$. Fix $i \in\{1, \ldots, d\}$. For the sequel it is convenient to express the kernel of $\tilde{\mathcal{R}}_{\alpha, i}$ with respect to the polynomial measure $m_{\alpha}$ on $\mathbf{R}_{+}^{d}$, defined by

$$
\mathrm{d} m_{\alpha}(x)=e^{|x|^{2}} \mathrm{~d} \tilde{\mu}_{\alpha}(x) .
$$

So we obtain that, for $x \notin \operatorname{supt} f$,

$$
\tilde{\mathcal{R}}_{\alpha} f(x)=\int_{\mathbf{R}_{+}^{d}} \mathcal{K}(x, y) f(y) \mathrm{d} m_{\alpha}(y),
$$

with $\mathcal{K}(x, y)=\nabla K_{1 / 2}\left(x^{2}, y^{2}\right) e^{|y|^{2}}$. In particular $\mathcal{K}_{i}(x, y)=\int_{[-1,1]^{d}} \mathcal{K}_{i}(x, y, s) \Pi_{\alpha}(s) \mathrm{d} s$ with

$$
\mathcal{K}_{i}(x, y, s)=C \int_{0}^{1}\left(\log \frac{1}{r}\right)^{-1 / 2}(1-r)^{-|\alpha|-d} \frac{-\sqrt{r}}{(1-r)}\left(\sqrt{r} x_{i}-y_{i} s_{i}\right) e^{-\left(q_{-}\left(r x^{2}, y^{2}, s\right)\right) /(1-r)} \frac{\mathrm{d} r}{r} .
$$

Observe that the whole reasoning contained therein goes roughly along lines of the proof of the main theorem in [12].

In order to define the "local" region, we consider the extra variable $s$ in $[-1,1]^{d}$. This artifice is suggested by the description of the local region in polar coordinates in the Ornstein-Uhlenbeck case, and by the form of the kernel $\mathcal{K}$. So the local region $N_{\tau}$ is the set

$$
\left\{(x, y, s) \in \mathbf{R}_{+}^{d} \times \mathbf{R}_{+}^{d} \times[-1,1]^{d}: q_{-}\left(x^{2}, y^{2}, s\right)^{1 / 2} \leqslant \frac{(8 \alpha+8) \tau}{1+|x|+|y|}\right\} .
$$

Indeed, for half-integer value of the multi-index $\alpha, N_{\tau}$ is equivalent to polyradial expression of the local region introduced for the Ornstein-Uhlenbeck semigroup in $[\mathbf{8}, \mathbf{9}]$.

Now we shall decompose $\widetilde{\mathcal{R}}_{\alpha, i}$ into a "global" and "local" parts. Let $\varphi$ be a cut-off function on $\mathbf{R}_{+}^{d} \times \mathbf{R}_{+}^{d} \times[-1,1]^{d}$ such that $0 \leqslant \varphi \leqslant 1$,

$$
\varphi(x, y, s)= \begin{cases}1, & (x, y, s) \in N_{1}, \\ 0, & (x, y, s) \notin N_{2},\end{cases}
$$

and

$$
\left|\nabla_{x} \varphi(x, y, s)\right|+\left|\nabla_{y} \varphi(x, y, s)\right|<\frac{c}{q_{-}\left(x^{2}, y^{2}, s\right)^{1 / 2}}
$$

Define

$$
\begin{aligned}
\tilde{\mathcal{R}}_{i}^{g l o b} f(x) & =\int_{\mathbf{R}_{+}^{d}} \int_{[-1,1]^{d}} \mathcal{K}_{i}(x, y, s)(1-\varphi(x, y, s)) \mathrm{d} s f(y) \mathrm{d} m_{\alpha}(y), \\
\tilde{\mathcal{R}}_{i}^{l o c} f & =\widetilde{\mathcal{R}}_{\alpha, i} f-\tilde{\mathcal{R}}_{i}^{\text {glob }} f,
\end{aligned}
$$

on bounded measurable functions $f$. The boundedness of these operators implies the weak type $(1,1)$ of $\widetilde{\mathcal{R}}_{\alpha}$. First we study $\widetilde{\mathcal{R}}_{i}^{\text {glob }}$. 
PRoposition 3.1. The operator $\widetilde{\mathcal{R}}_{i}^{\text {glob }}$ is of weak type $(1,1)$ with respect to the measure $\tilde{\mu}_{\alpha}$.

Proof: Since $((1-r) / \log (1 / r))^{1 / 2}$ is bounded for $r \in(0,1)$, after the change of variables $t=1-r$, we can control $\widetilde{\mathcal{R}}_{i}^{\text {glob }}$, by the operator

$$
f \mapsto \int_{\mathbf{R}_{+}^{d}} \int_{[-1,1]^{d}} \mathcal{K}^{\prime}(x, y, s) \Pi_{\alpha}(s) \mathrm{d} s f(y) \mathrm{d} m_{\alpha}(y),
$$

with

$$
\mathcal{K}^{\prime}(x, y, s)=\int_{0}^{1} t^{-|\alpha|-d-1}\left(\frac{q_{-}\left((1-t) x^{2}, y^{2}, s\right)}{t}\right)^{1 / 2} e^{-\left(q_{-}\left((1-t) x^{2}, y^{2}, s\right)\right) / t}(1-\varphi(x, y, s)) \frac{\mathrm{d} t}{\sqrt{1-t}} .
$$

We now proceed with the estimate of the kernel $\mathcal{K}^{\prime}(x, y, s)$ in terms of $\overline{\mathcal{K}}(x, y, s)$, defined by

$$
\begin{aligned}
& \overline{\mathcal{K}}(x, y, s) \\
& \quad= \begin{cases}e^{-|y|^{2},} & \sum_{i=1}^{d} x_{i} y_{i} s_{i} \leqslant 0 \\
\left(\frac{q_{+}\left(x^{2}, y^{2}, s\right)}{q_{-}\left(x^{2}, y^{2}, s\right)}\right)^{(|\alpha|+d) / 2} e^{-\left(|y|^{2}-|x|^{2}\right) / 2-\left(q_{-}\left(x^{2}, y^{2}, s\right)^{1 / 2} q_{+}\left(x^{2}, y^{2}, s\right)^{1 / 2}\right) / 2}, & \text { otherwise. }\end{cases}
\end{aligned}
$$

The proof is a simple modification to our case of the arguments used in the proof of Proposition 2.2 in [9] and we omit the details. Now by an adaptation of [8, Proposition 2.1] and by $\left[11\right.$, Theorem 4.3 , Remark 4.6], the weak type $(1,1)$ boundedness of $\tilde{\mathcal{R}}_{i}^{\text {glob }}$ follows from these estimates.

REMARK 3.2. Since the operator

$$
f \mapsto \int_{\mathbf{R}_{+}^{d}} \int_{[-1,1]^{d}} \overline{\mathcal{K}}(x, y, s) \Pi_{\alpha}(s) \mathrm{d} s f(y) \mathrm{d} m_{\alpha}(y)
$$

is bounded on $L^{2}\left(\tilde{\mu}_{\alpha}\right)$ (see [12, Proposition 3]), we may deduce that $\widetilde{\mathcal{R}}_{i}^{\text {glob }}$ is bounded on $L^{2}\left(\tilde{\mu}_{\alpha}\right)$. In the following, we need the $L^{2}\left(\tilde{\mu}_{\alpha}\right)$-boundeness of $\tilde{\mathcal{R}}_{i}^{\text {glob }}$ to study $\tilde{\mathcal{R}}_{i}^{\text {loc }}$.

Now to prove our main result, it is enough to get the weak type $(1,1)$ estimate for $\tilde{\mathcal{R}}_{i}^{\text {loc }}$. Observe that the operator $\tilde{\mathcal{R}}_{i}^{\text {loc }}$ is a singular integral operator. Moreover, outside the diagonal, its kernel

$$
\begin{aligned}
& \mathcal{K}_{i}^{l o c}(x, y, s) \\
& =C \int_{0}^{1}\left(\log \frac{1}{r}\right)^{-1 / 2}(1-r)^{-|\alpha|-d-1}(-\sqrt{r})\left(\sqrt{r} x_{i}-y_{i} s_{i}\right) e^{-\left(q_{-}\left(r x^{2}, y^{2}, s\right)\right) /(1-r)} \frac{\mathrm{d} r}{r} \varphi(x, y, s),
\end{aligned}
$$

satisfies suitable gradient estimates.

LEMMA 3.3. There exists a constant $C$ such that

$$
\begin{aligned}
\left|\mathcal{K}_{i}^{l o c}(x, y, s)\right| & \leqslant C q_{-}\left(x^{2}, y^{2}, s\right)^{-|\alpha|-d}, \\
\left|\nabla_{(x, y)} \mathcal{K}_{i}^{l o c}(x, y, s)\right| & \leqslant C q_{-}\left(x^{2}, y^{2}, s\right)^{-|\alpha|-d-1 / 2},
\end{aligned}
$$

whenever $(x, y, s) \in N_{t}$, for each $t \in \mathbf{R}_{+}$. 
Proof: We split the kernel $\mathcal{K}^{\text {loc }}(x, y, s)$ into two integrals

$$
\mathcal{K}_{i}^{l o c}(x, y, s)=\int_{0}^{1 / 2} \cdots \mathrm{d} r+\int_{1 / 2}^{1} \cdots \mathrm{d} r
$$

Since

$$
\begin{aligned}
\frac{\left|\sqrt{r} x_{i}-y_{i} s_{i}\right|}{\sqrt{1-r}} e^{-\left(\sqrt{\left.q_{-}\left(r x^{2}, y^{2}, s\right) /(1-r)\right)}\right.} & \leqslant \frac{q_{-}\left(r x^{2}, y^{2}, s\right)}{\sqrt{1-r}} e^{-\left(q_{-}\left(r x^{2}, y^{2}, s\right)\right) /(1-r)} \\
& \leqslant C e^{-\left(q_{-}\left(r x^{2}, y^{2}, s\right)\right) /(2(1-r))}
\end{aligned}
$$

for $(x, y, s) \in N_{t}$ and $0<r<1$

$$
\left|\frac{\sqrt{r} x_{i}-y_{i} s_{i}}{\sqrt{1-r}}\right| e^{-\left(q_{-}\left(r x^{2}, y^{2}, s\right)\right) /(1-r)} \leqslant C e^{-\left(q_{-}\left(x^{2}, y^{2}, s\right)\right) /(c(1-r))},
$$

where the last inequality is a consequence of the following estimate true in $N_{\tau}$

$$
q_{-}\left(r x^{2}, y^{2}, s\right) \geqslant q_{-}\left(x^{2}, y^{2}, s\right)-2 C\left(1-r^{1 / 2}\right)
$$
(see $[12,(2.6)])$. Now it is easy to see that the integral $\int_{0}^{1 / 2} \cdots \mathrm{d} r \leqslant C$. On the other
hand

$$
\begin{aligned}
\int_{1 / 2}^{1} \cdots \mathrm{d} r & \leqslant C \int_{1 / 2}^{1}(1-r)^{-|\alpha|-d} e^{-\left(q_{-}\left(x^{2}, y^{2}, s\right)\right) /(c(1-r))} \mathrm{d} r \\
& \leqslant q_{-}\left(x^{2}, y^{2}, s\right)^{-|\alpha|-d}
\end{aligned}
$$

as required. To complete the proof, it remains to prove the gradient estimates. We have that, for each $j=1, \ldots, d$

$$
\begin{aligned}
& \left|\partial_{x_{j}} \mathcal{K}_{i}^{l o c}(x, y, s)\right| \\
& \leqslant\left|\mathcal{K}_{i}(x, y, s) \partial_{x_{j}} \varphi(x, y, s)\right| \\
& \quad+\delta_{i j} C\left|\int_{0}^{1}\left(\log \frac{1}{r}\right)^{-1 / 2}(1-r)^{-|\alpha|-d-1} e^{-\left(q-\left(r x^{2}, y^{2}, s\right)\right) /(1-r)} \mathrm{d} r\right| \\
& \quad+C\left|\int_{0}^{1}\left(\log \frac{1}{r}\right)^{-1 / 2}(1-r)^{-|\alpha|-d-2}\left(\sqrt{r} x_{i}-y_{i} s_{i}\right)\left(\sqrt{r} x_{j}-y_{j} s_{j}\right) e^{-\left(q-\left(r x^{2}, y^{2}, s\right)\right) /(1-r)} \mathrm{d} r\right| .
\end{aligned}
$$

Using (8) and arguing as in the first part of this proof, it is a simple matter to verify that these integrals are controlled by $C q_{-}\left(x^{2}, y^{2}, s\right)^{-|a|-d-1 / 2}$. Similarly, the same estimates hold for $\left|\partial_{y_{j}} \mathcal{K}^{l o c}(x, y, s)\right|$ and this concludes the proof.

However, $\left(\mathbf{R}_{+}^{d}, \tilde{\mu}_{\alpha}\right)$ is not a space of homogeneous type in the sense of $[1]$, because the measure $\tilde{\mu}_{\alpha}$ is not a doubling measure. Therefore we cannot apply directly the Calderón-Zygmund theory to the operator $\tilde{\mathcal{R}}_{i}^{\text {loc }}$, to prove that it is bounded from $L^{1}\left(\tilde{\mu}_{\alpha}\right)$ to $L^{1, \infty}\left(\tilde{\mu}_{\alpha}\right)$. 
Nevertheless, we shall see that the problem may be reduced to studying the operator on $L^{1}\left(m_{\alpha}\right)$, where $m_{\alpha}$ is the measure defined in (7). Indeed, $\mathbf{R}_{+}^{d}$ with the measure $m_{\alpha}$ and the Euclidean distance is a space of homogeneous type. To pass from $\left(\mathbf{R}_{+}^{d}, \tilde{\mu}_{\alpha}\right)$ to $\left(\mathbf{R}_{+}^{d}, m_{\alpha}\right)$, we need to observe that there exists a collection of balls $\left\{B_{j}\right\}_{j \in N}$, covering $\mathbf{R}_{+}^{d}$, such that the collection $\left\{\delta B_{j}\right\}_{j \in \mathbf{N}}$ has bounded overlap for each $\delta>1$ and on $B_{j}$ the measure $\tilde{\mu}_{\alpha}$ is equivalent to the measure $m_{\alpha}$ (see [10, Lemma 4]), that is, there exist $C_{0}, C_{1}>0$, such that for every measurable subset $E$ of $B_{j}$

$$
C_{0} e^{-\left|x_{j}\right|^{2}} m_{\alpha}(E) \leqslant \tilde{\mu}_{\alpha}(E) \leqslant C_{1} e^{-\left|x_{j}\right|^{2}} m_{\alpha}(E) .
$$

REMARK 3.4. Let $\mathcal{T}$ be an operator mapping bounded measurable functions with compact support to locally integrable functions. For each $\delta>1$ fixed, define

$$
\mathcal{T}_{\ell} f=\sum_{j} \chi_{B_{j}} \mathcal{T}\left(f \chi_{\delta B_{j}}\right)
$$

for each bounded measurable function $f$. We have that if $\mathcal{T}$ extends to a bounded operator on $L^{p}\left(\tilde{\mu}_{\alpha}\right)$ or on $L^{p}\left(m_{\alpha}\right)$ for some $p \in[1, \infty)$, then $\mathcal{T}_{\ell}$ extends to a bounded operator on $L^{p}\left(\tilde{\mu}_{\alpha}\right)$ and on $L^{p}\left(m_{\alpha}\right)$. The $L^{p}$-operator norm of $\mathcal{T}_{\ell}$ with respect to both measures, is bounded by a constant times the $L^{p}$-operator norm of $\mathcal{T}$. Moreover the same result holds for the weak type estimates $(p, p)$, with $p \in[1, \infty)$ (see $[10$, Lemma 5]).

Now we can prove the weak type $(1,1)$ boundeness of $\tilde{\mathcal{R}}_{\alpha}$.

THEOREM 3.5. The first order Riesz-Laguerre transforms $\widetilde{\mathcal{R}}_{\alpha, i}$ are of weak type $(1,1)$ with respect to the measure $\tilde{\mu}_{\alpha}$.

Proof: By Proposition 3.1, $\widetilde{\mathcal{R}}_{i}^{\text {glob }}$ is of weak type $(1,1)$. Thus by $(9)$, it is enough to analyse the operator $\tilde{\mathcal{R}}_{i}^{\text {loc }}$. Let $\left\{B_{j}\right\}_{j \in N}$ be the family of balls defined above. We shall fix $\delta>1$ later. We may write $\widetilde{\mathcal{R}}_{i}^{\text {loc }}$ as

$$
\tilde{\mathcal{R}}_{i}^{l o c} f=\tilde{\mathcal{R}}_{i}^{l o c}\left(f \chi_{\delta B_{j}}\right)+\tilde{\mathcal{R}}_{i}^{l o c}\left(\left(1-\chi_{\delta B_{j}}\right) f\right) .
$$

By multiplying by $\chi_{B_{j}}$ and adding over $j$, we get

$$
\begin{aligned}
\left|\tilde{\mathcal{R}}_{i}^{l o c} f\right| & \leqslant\left|\sum_{j} \chi_{B_{j}} \tilde{\mathcal{R}}_{i}^{l o c}\left(f \chi_{\delta B_{j}}\right)\right|+\left|\sum_{j} \chi_{B_{j}} \widetilde{\mathcal{R}}_{i}^{l o c}\left(\left(1-\chi_{\delta B_{j}}\right) f\right)\right| \\
& :=\left|\mathcal{R}_{\ell}^{l o c} f\right|+|R f| .
\end{aligned}
$$

By [7] and Remark $3.2, \tilde{\mathcal{R}}_{\alpha}$ and $\tilde{\mathcal{R}}_{i}^{\text {glob }}$ are bounded on $L^{2}\left(\widetilde{\mu}_{\alpha}\right)$. It follows that $\tilde{\mathcal{R}}_{i}^{\text {loc }}$ is bounded on $L^{2}\left(\tilde{\mu}_{\alpha}\right)$. So by Remark $3.4, \mathcal{R}_{\ell}^{\text {loc }}$ is bounded on $L^{2}$ with respect to both measures, $m_{\alpha}$ and $\tilde{\mu}_{\alpha}$. We know that $R$ is bounded both on $L^{p}\left(\tilde{\mu}_{\alpha}\right)$ and on $L^{p}\left(m_{\alpha}\right)$, for $1 \leqslant p \leqslant \infty$ (see Proposition $6(19)$ of $[10]$ ). Thus the operator $\tilde{\mathcal{R}}_{i}^{\text {loc }}$ is a singular integral operator, whose kernel is $\int_{[-1,1]^{d}} \mathcal{K}(x, y, s) \varphi(x, y, s) \Pi_{\alpha}(s) \mathrm{d} s$, outside the diagonal. By the claim, $\widetilde{\mathcal{R}}_{i}^{\text {loc }}$ is bounded on $L^{2}\left(m_{\alpha}\right)$. Moreover, by (8) and Lemma 3.3

$$
\left|\nabla_{(x, y)}[\mathcal{K}(x, y, s) \varphi(x, y, s)]\right| \leqslant C q_{-}\left(x^{2}, y^{2}, s\right)^{-|\alpha|-d-1 / 2}
$$


for each $(x, y, s) \in N_{2}$ and $x \neq y$. Hence by an extension of the classical CalderónZygmund theory for a singular operator on $\left(\mathbf{R}_{+}^{d}, m_{a}\right)$ (see for the one-dimensional case [10, Proposition 9]), $\tilde{\mathcal{R}}_{i}^{\text {loc }}$ is of weak type $(1,1)$ with respect to the measure $m_{\alpha}$. By Remark $3.4, \mathcal{R}_{\ell}^{\text {loc }}$ is of weak type $(1,1)$ with respect to the measure $\tilde{\mu}_{\alpha}$ and

$$
\left\|\tilde{\mathcal{R}}_{i}^{l o c} f\right\|_{L^{1, \infty}\left(\tilde{\mu}_{\alpha}\right)} \leqslant\left\|\mathcal{R}_{\ell}^{l o c} f\right\|_{L^{1, \infty}\left(\tilde{\mu}_{\alpha}\right)}+\|R f\|_{L^{1}\left(\tilde{\mu}_{\alpha}\right)},
$$

as desired.

REMARK 3.6. Now Theorem 1.1 follows easily by the weak type $(1,1)$ of $\widetilde{\mathcal{R}}_{\alpha}$ with respect to the measure $\tilde{\mu}_{\alpha}$, proved in Theorem 3.5.

\section{A COUNTEREXAMPLE FOR OPERATORS OF ORDER AT LEAST THREE}

In this section, we present a counterexample to show that the Riesz transform of order at least three are not of weak type $(1,1)$ with respect the Laguerre measure. For any multi-index $a \in \mathbf{R}_{+}^{d}$, let $\mathcal{R}_{\alpha}^{a}$ be the Riesz-Laguerre transform of order $a$, defined by

$$
\mathcal{R}_{\alpha}^{a}=\nabla_{\alpha}^{a} \mathcal{L}_{\alpha}^{-|a| / 2} \mathcal{P}_{0}^{\alpha \perp}
$$

As in Section 3, we may reduce the problem to the analysis of $\widetilde{\mathcal{R}}_{\alpha}^{a}=\mathcal{U}_{\Psi} \mathcal{R}_{\alpha}^{a} \mathcal{U}_{\Psi}^{-1}$. By (4), it is quite simple to see that $\partial^{a} \tilde{\mathcal{L}}_{\alpha}^{-|a| / 2} \widetilde{\mathcal{P}}_{0}^{\alpha \perp}$ is given by a singular integral operator and, off the diagonal, its kernel with respect to the measure $m_{\alpha}$ is

$$
\mathcal{K}^{\alpha}(x, y)=C \int_{[-1,1]^{d}} \mathcal{K}^{a}(x, y, s) \Pi_{\alpha}(s) \mathrm{d} s,
$$

with

$$
\begin{aligned}
\mathcal{K}^{a}(x, y, s)=\int_{0}^{1}\left(\log \frac{1}{r}\right)^{|a| /(2-1)}(1-r)^{-|a|-d} \frac{(-\sqrt{r})^{|a|}}{(1-r)^{|a| / 2}} \\
\prod_{i=1}^{d} H_{a_{i}}\left(\frac{\sqrt{r_{i}} x_{i}-y_{i} s_{i}}{\sqrt{1-r}}\right) e^{-\left(q-\left(\sqrt{r} x^{2}, y^{2}, s\right)\right) /(1-r)} \frac{\mathrm{d} r}{r},
\end{aligned}
$$

where $H_{a_{i}}$ is the Hermite polynomial of degree $a_{i}$.

THEOREM 4.1. Let $|\alpha| \geqslant 3$. Then the operator $\partial^{a} \widetilde{\mathcal{L}}_{\alpha}^{-|a| / 2} \mathcal{P}_{0}^{\alpha \perp}$ is not of weak type $(1,1)$ with respect to the measure $\tilde{\mu}_{\alpha}$.

PROOF: For $\eta \in \mathbf{R}_{+}^{d}$ fixed, with $\eta_{i}=|\eta| / \sqrt{d}$ for each $i=1, \ldots, d$ and $|\eta|$ sufficiently large, we want to estimate the kernel $\mathcal{K}^{a}(x, \eta)$, whenever $x$ is in $J=\{\xi(\eta) /|\eta|+v: v \perp$ $\eta,|v|<1,|\eta| / 2<\xi<(3 / 4)|\eta|\}$. We claim that for these choices of $x$ and $\eta$ there exists a constant $\mathrm{C}$ such that,

$$
\mathcal{K}^{a}(x, \eta) \geqslant C \eta^{|a|-2|\alpha|-d-1} e^{\xi^{2}-\eta^{2}} .
$$


Assuming this claim for the moment, we find a counterexample for the $(1,1)$ boundedness of the Riesz-Laguerre transform of order al least 3. Indeed, let $f>0$ in $L^{1}\left(\tilde{\mu}_{\alpha}\right)$ be a close approximation of a point mass at $\eta$, with $\|f\|_{L^{1}\left(\tilde{\mu}_{\alpha}\right)}=1$. Then $\partial^{a} \tilde{\mathcal{L}}_{\alpha}^{-|a| / 2} \mathcal{P}_{0}^{\alpha \perp} f(x)$ will be close to $e^{\eta} \mathcal{K}_{a}(x, \eta)$. By (11) if $x \in J$, we get that $e^{\eta} \mathcal{K}_{a}(x, \eta) \geqslant C \eta^{a-2 \alpha-2} e^{(\eta / 2)^{2}}$. Now we suppose that $\partial^{\alpha} \widetilde{\mathcal{L}}_{\alpha}^{-|a| / 2} \tilde{\mathcal{P}}_{0}^{\alpha \perp}$ is of weak type $(1,1)$ with respect to the measure $\tilde{\mu}_{\alpha}$, then there exists a constant $C$ such that

$$
\tilde{\mu}_{\alpha}\left\{J_{x}\right\} \leqslant \tilde{\mu}_{\alpha}\left\{x \in \mathbf{R}_{+}: \partial^{a} \tilde{\mathcal{L}}_{\alpha}^{-|a| / 2} \tilde{\mathcal{P}}_{0}^{\alpha \perp}(x)>\lambda\right\} \leqslant C \lambda^{-1},
$$

with $\lambda=c \eta^{|a|-2|a| \mid-d-1} e^{(\eta / 2)^{2}}$. Since $x_{i} \geqslant C|\eta|$ for each $i=1, \ldots, d$ and $\tilde{\mu}_{\alpha}\{J\}$ $\geqslant C e^{-(\eta / 2)^{2}} \eta^{2|\alpha|+d-1}$, letting $\eta$ tend to infinity, we find a contradiction whenever $|a|>2$.

Now in order to show the claim (11), we split the kernel in three parts, corresponding to the decomposition of $[-1,1]^{d}$ in $I_{1} \cup I_{2} \cup I_{3}$, with

$$
\begin{aligned}
& I_{1}=\left[1-\frac{1}{|\eta|^{2}}, 1\right]^{d} \\
& I_{2}=\left[1-\frac{1}{|\eta|^{d}}, 1\right]^{d} \backslash I_{1} \\
& I_{3}=\left(I_{1} \cup I_{2}\right)^{c},
\end{aligned}
$$

that is, $\mathcal{K}_{a}(x, y)=k_{1}(x, y)+k_{2}(x, y)+k_{3}(x, y)$ where $k_{i}(x, y)=\int_{I_{i}} \mathcal{K}_{a}(x, y, s) \Pi_{\alpha}(s) \mathrm{d} s$, with $i=1,2,3$. In particular, we estimate $\mathcal{K}_{a}(x, \eta)$ whenever $x \in J$ by the estimates of $k_{1}(x, \eta), k_{2}(x, \eta)$ (from below) and $k_{3}(x, \eta)$ (from above).

Observe that if $s \in I_{1} \cup I_{2}$,

$$
-\frac{\sqrt{r} x_{i}-y_{i} s_{i}}{\sqrt{1-r}} \geqslant c|\eta|
$$

then

$$
(-1)^{a_{i}} H_{a_{i}}\left(\frac{\sqrt{r} x_{i}-y_{i} s_{i}}{\sqrt{1-r}}\right)>c \eta^{|a|} .
$$

So the integrand $\mathcal{K}_{a}(x, \eta, s)$ is positive for $x \in J, s \in I_{1} \cup I_{2}$ and $0<r<1$. Moreover, if $x \in J$ we have that

$$
\begin{aligned}
& q_{-}\left(r x^{2}, \eta^{2}, s\right)=r|x|^{2}+|\eta|^{2}-2 \sqrt{r} \sum_{i=1}^{d} x_{i} \eta_{i} s_{i} \\
& \leqslant(r-1)\left(\xi^{2}-|\eta|^{2}\right)+(\xi-\sqrt{r}|\eta|)^{2}+c \quad s \in I_{1}, \\
& \leqslant(r-1)\left(\xi^{2}-|\eta|^{2}\right\rangle+(\xi-\sqrt{r}|\eta|)^{2}+c|\eta| \quad s \in I_{2} \text {. }
\end{aligned}
$$

In particular, for $1 / 16<r<9 / 16$

$$
\begin{array}{rlrl}
e^{-\left(q-\left(\sqrt{r} x^{2}, \eta^{2}, s\right)\right) /(1-r)} & \geqslant C e^{\xi^{2}-\eta^{2}} e^{-c(\xi-\sqrt{r} \eta)^{2}} & s \in I_{1}, \\
& \geqslant C e^{\xi^{2}-\eta^{2}} e^{-c(\xi-\sqrt{r} \eta)^{2}-c|\eta|} & s \in I_{2} .
\end{array}
$$


Moreover it is quite simple to see that

$$
\begin{aligned}
\int_{I_{1}} \Pi_{\alpha}(s) \mathrm{d} s & \geqslant C|\eta|^{-2|\alpha|-d}, \\
\int_{1 / 16}^{9 / 16} e^{-c(\xi-\sqrt{r} \eta)^{2}} \mathrm{~d} r & \geqslant C|\eta|^{-1} .
\end{aligned}
$$

So the previous estimates imply that for $x \in J$

$$
\begin{aligned}
e^{|\eta|}\left(k_{1}(x, \eta)+k_{2}(x, \eta)\right. & \geqslant e^{|\eta|} k_{1}(x, \eta) \\
& \geqslant C \eta^{|a|-2|\alpha|-d-1} e^{\xi^{2}}\left(1+c e^{-|\eta|}\right) \\
& \geqslant C \eta^{|a|-2|\alpha|-d-1} e^{\xi^{2}} .
\end{aligned}
$$

Now we want to control $k_{3}(x, \eta)$, whenever $x \in J$. To that end we use that over the global region, each of the kernels $\mathcal{K}_{a}(x, y, s)$ is pointwise controlled by the function

$$
\left(q_{+}\left(x^{2}, y^{2}, s\right) q_{-}\left(x^{2}, y^{2}, s\right)\right)^{(|a|-2) / 4}\left(\left(q_{+}\left(x^{2}, y^{2}, s\right) q_{-}\left(x^{2}, y^{2}, s\right)\right)^{1 / 4} \frac{|x||\eta|}{|x|^{2}+|\eta|^{2}}+1\right) \overline{\mathcal{K}}(x, y, s) .
$$

These estimates follow by a simple adaptation to our case of the corresponding estimates in [3]. Now let $Q_{ \pm}=q_{ \pm}\left(x^{2}, \eta^{2}, s\right)$ and $\cos \theta=\left(\sum_{i=1}^{d} x_{i} \eta_{i} s_{i}\right) /(|x||\eta|)$, whenever $(x, s)$ $\in J \times I_{3}$. It is quite simple to verify that there exists a constant $C$ such that $|\eta|^{2} \sin ^{2} \theta$ $\geqslant C \eta$. Thus, since $Q_{-} Q_{+} \leqslant 4|\eta|^{4}$ and $Q_{-} \geqslant|x|^{2} \sin ^{2} \theta$, we obtain that $(x, \eta, s)$ is in the global region and

$$
\begin{aligned}
& e^{|\eta|^{2}}\left|k_{3}(x, \eta)\right| \\
& \quad \leqslant\left(Q_{+} Q_{-}\right)^{(|a|-2) / 4}\left(\left(Q_{+} Q_{-}\right)^{1 / 4} \frac{|x||\eta|}{|x|^{2}+|\eta|^{2}}+1\right)\left(\frac{Q_{+}}{Q_{-}}\right)^{(|\alpha|+d) / 2} e^{\left(|\eta|^{2}-|x|^{2}-\sqrt{\left.q_{+}+-\right) / 2} e^{|x|^{2}}\right.}
\end{aligned}
$$

and so

$$
\begin{aligned}
e^{|\eta|^{2}}\left|k_{3}(x, \eta)\right| & \leqslant C|\eta|^{|\alpha|-1}\left(\frac{|\eta|^{2}}{Q_{-} Q_{+}}\right)^{(\mid \alpha+d) / 2} e^{-\left(\sin ^{2} \theta|x|^{2}|\eta|^{2}\right) /\left(\sqrt{\left.Q_{-} Q_{+}\right)}\right.} e^{|x|^{2}} \\
& \leqslant C|\eta|^{|a|-1-|\alpha|-d} e^{-c|\eta|} e^{\xi^{2}}
\end{aligned}
$$

By (12) and (13), we may estimate from above $\mathcal{K}_{a}(x, \eta)$. Indeed we have that

$$
\begin{aligned}
\mathcal{K}_{a}(x, \eta) & \geqslant k_{1}(x, \eta)+k_{2}(x, \eta)-\left|k_{3}(x, \eta)\right| \\
& \geqslant C e^{\xi}|\eta|^{|a|-2|\alpha|-d-1}\left(1-c|\eta|^{\alpha} e^{-c|\eta|}\right) \\
& \geqslant C e^{|\eta|^{2} / 2}|\eta|^{|a|-2|\alpha|-d-1},
\end{aligned}
$$

for $|\eta|$ sufficiently large. This concludes the proof of the claim and of the Theorem. 


\section{REFERENCES}

[1] R.R. Coifman and G. Weiss, Analyse harmonique non-commutative sur certains espaces homogènes, Lecture Notes in Mathématiques 242 (Springer-Verlag, Berlin, New York, 1971).

[2] U. Dinger, 'Weak Type $(1,1)$ Estimates of the maximal function for the Laguerre semigroup in finite dimensions', Rev. Mat. Iberoamericana 8 (1992), 93-118.

[3] J. Garcia-Cuerva, G. Mauceri, P. Sjögren and J.L. Torrea, 'Higher-Order Riesz operators for Ornstein-Uhlenbeck semigroups', Potential Anal. 10 (1999), 379-407.

[4] P. Graczyk, J.J. Loeb, I. Lopez, A. Nowak and W. Urbina, 'Higher order Riesz transforms, fractional derivatives, and Sobolev spaces for Laguerre expansions', J. Math. Pures Appl. 84 (2005), 375-405.

[5] C. Gutiérrez, A. Incognito and J.L. Torrea, 'Riesz Transforms, g-functions and multipliers for Laguerre semigroup', Houston J. Math. 27 (2001), 579-592.

[6] B. Muckenhoupt, 'Conjugate functions for Laguerre expansions', Trans. Amer. Math. Soc. 139 (1969), 231-242.

[7] A. Nowak, 'On Riesz transforms for Laguerre expansions', J. Funct. Anal. 215 (2004), 217-240.

[8] T. Menárguez, S. Pérez and F. Soria, 'The Mehler maximal function: a geometric proof of the weak type 1', J. London Math. Soc. (2) 61 (2000), 846-856.

[9] S. Pérez and F. Soria, 'Operators associated with the Ornstein-Uhlenbeck semigroup', $J$. London Math. Soc. 61 (2000), 857-871.

[10] E. Sasso, 'Functional calculus for the Laguerre operator', Math. Z. 249 (2005), 683-711.

[11] E. Sasso, 'Maximal operators for the holomorphic Laguerre semigroup', Math. Scand. 97 (2005), 235-265.

[12] E. Sasso, 'Spectral multipliers of Laplace transform type for the Laguerre operator', Bull. Austral. Math. Soc. 69 (2004), 255-266.

[13] G. Szegö, Orthogonal polynomials, (Revised Edition) (Amer. Math. Soc., Providence, 1959).

Dipartimento di Matematica

Università di Genova

via Dodecanesco 35

16146 Genova

Italy

e-mail: sasso@dima.unige.it 